\title{
Usefulness of 320-Row Area Detector Computed Tomography for the Diagnosis of Cystic Falx Meningioma
}

\author{
Sadaharu Tabuchi Sadao Nakajima \\ Department of Neurosurgery, Tottori Prefectural Central Hospital, Tottori, Japan
}

\section{Key Words}

Cystic meningioma $\cdot 320$-row area detector computed tomography $\cdot 4$-dimensional computed tomography angiography · Computed tomography perfusion

\begin{abstract}
We present a case of cystic falx meningioma. Cystic meningioma is rare and not easy to diagnose preoperatively; it is often misdiagnosed as other tumors, including glial or metastatic tumors with cystic or necrotic changes. This study showed the potential impact of 320-row computed tomography (CT) on image-based diagnostic evaluation of cystic meningioma with special attention to the novel techniques of 4-dimensional CT angiography (4D-CTA) and CT whole-brain perfusion (CTP). 4D-CTA showed the arterial supply feeding the tumor and late enhancement of the tumor nodule, similar to that seen in meningioma by conventional angiography. CTP showed that the tumor had a higher cerebral blood flow and cerebral blood volume and a longer mean transit time than adjacent brain tissue. These findings were consistent with meningioma and reinforced the other imaging findings, resulting in the correct preoperative diagnosis. The new techniques available for 320-row CT can potentially be used to improve differential diagnosis and preoperative assessment of cystic tumors with nodules.

(c) 2013 S. Karger AG, Basel
\end{abstract}

\section{Introduction}

Meningiomas are uncommonly associated with cysts and account for $1.6-10 \%$ of intracranial meningiomas [1-3]. Furthermore, cystic meningioma is not easy to diagnose preoperatively and is often misdiagnosed as a cystic glioma or metastatic brain tumor. We

Sadaharu Tabuchi, MD, PhD

Department of Neurosurgery

Tottori Prefectural Central Hospital

730 Ezu, Tottori 680-0901 (Japan)

E-Mail tabuchis@pref.tottori.jp 
Tabuchi et al.: Usefulness of 320-Row Area Detector Computed Tomography for the Diagnosis of Cystic Falx Meningioma

describe a case of cystic falx meningioma that was accurately diagnosed preoperatively by using new imaging techniques based on 320-row computed tomography (CT).

\section{Case Report}

A 52-year-old male had gradually developing motor weakness in his left leg and focal seizures. He came to our outpatient service and CT showed a large cystic lesion of the right frontal lobe with a small nodule adjacent to the falx. On admission, he was alert but had left hemiparesis. Magnetic resonance imaging (MRI) showed a huge cyst in the left frontal lobe that was compressing the motor cortex (fig. 1a). A round mass with a diameter of $2 \mathrm{~cm}$ was located at the falx and appeared to be a mural module. It was highly enhanced together with its attached dura mater, but the cyst wall was not enhanced (fig. 1b, c). Cystic falx meningioma, cystic glioma or dural metastasis with cyst was suspected. Screening of the entire body showed no cancer. The results of MR spectroscopy (high choline peak, very low level of creatine and $\mathrm{N}$-acetylaspartate) were consistent with an extra-axial tumor, such as a meningioma. Conventional cerebral angiography was not done. Additional imaging was performed using a 320-row CT scanner (Aquilion One, Toshiba Medical Systems, Nasu, Japan) that had a detector width of $160 \mathrm{~mm}$ and 320 detector rows. Time-resolved wholehead 4-dimensional CT angiography (4D-CTA) was performed instead of conventional digital subtraction angiography (DSA). The serial images showed the arterial supply feeding the tumor, and the tumor contrast became more pronounced at later time points, which indicated tumor staining commonly seen in meningioma (fig. 2).

After 4D-CTA, CT perfusion (CTP) imaging was performed and analyzed. The cerebral blood flow (CBF) in the tumor area $(53.0 \mathrm{ml} / \mathrm{min} / 100 \mathrm{~g})$ was higher than that in the adjacent brain tissue $(30.1 \mathrm{ml} / \mathrm{min} / 100 \mathrm{~g}$ ) (fig. 3a). The cerebral blood volume (CBV) in the tumor $(6.4 \mathrm{ml} / 100 \mathrm{~g})$ was also higher than that in the adjacent brain tissue $(2.3 \mathrm{ml} / 100 \mathrm{~g})$ (fig. $3 \mathrm{~b})$. Furthermore, the mean transit time (MTT) of the tumor (7.2 s) was longer than that of the adjacent brain tissue ( $4.9 \mathrm{~s}$ ) (fig. 3c). The perfusion parameters of this tumor suggested that the tumor blood flow and capillary blood volume were 176 and $278 \%$ of the adjacent brain tissue, respectively. The possibility of congestion in the capillary bed of the tumor was suggested by the longer MTT. These findings were consistent with the data of meningioma obtained in our department [unpubl. observation].

As all of the preoperative imaging findings were consistent with meningioma, we made a definitive preoperative diagnosis of cystic falx meningioma. After the cyst fluid was aspirated during surgery, a reddish tumor showing the typical appearance of meningioma was found arising from the falx. The tumor was totally removed along with its attachment (Simpson grade 1). The histological diagnosis was meningothelial meningioma (WHO grade 1). Intraoperative frozen sections revealed no tumor cells in the cyst wall. According to the preoperative imaging and intraoperative findings, we regarded this case as an extratumoral cyst, classified as Nauta type 3 [4]. The cyst wall was left without excision except for the part attached to it. Postoperative MRI showed the absence of the tumor and collapse of the cyst. The patient's postoperative course was uneventful, and he was discharged from the hospital free of all symptoms. 
Tabuchi et al.: Usefulness of 320-Row Area Detector Computed Tomography for the Diagnosis of Cystic Falx Meningioma

\section{Discussion}

Meningiomas are generally solid tumors, and their classical appearance on CT and MRI usually leads to a correct diagnosis. A problem with the diagnosis arises when meningiomas have cystic components that can be confused with other tumors, including glial or metastatic tumors with cystic or necrotic changes. The accuracy of the differential diagnosis strongly affects preoperative surgical planning.

Time-resolved whole-head 4D-CTA using 320-row CT has recently been performed as part of the diagnostic work-up for arteriovenous malformations [5]. Catheter-based DSA is time consuming and carries a rather high incidence of silent embolic events and a small risk of transient or permanent neurologic deterioration. Therefore, noninvasive alternatives to angiography are of interest. It is conceivable that imaging methods based on 320-row CT may replace DSA in cases that require time-resolved imaging. Until now, 4D-CTA has only been used to diagnose cerebrovascular disorders. To our knowledge, there are no reports in the English literature of the use of this method for the diagnosis of brain tumors. In our case, 4D-CTA was helpful for tumor evaluation and preoperative planning. Additional studies using this method are needed to evaluate various brain tumors.

Perfusion imaging techniques provide additional information regarding tumor hemodynamics. Traditionally, perfusion imaging of brain tumors has been performed with MRI. However, CTP provides a linear relationship between tissue attenuation and the tissue concentration of contrast agent, which allows the reliable quantitative measurement of CBF and CBV. Because of wide availability, faster scanning times and low cost, CTP is potentially well suited for brain tumor assessment [6].

Generally, the differentiation between cystic meningioma and intra-axial tumors is difficult. Meningiomas are generally highly vascularized tumors, and the CBV ratios obtained by perfusion MRI are higher than the CBV ratios of intra-axial tumors [7]. To our knowledge, there are no previous reports describing the CTP characteristics of cystic meningiomas. Accumulation of perfusion patterns of meningiomas and intra-axial tumors should lead to the further development of CTP as a useful tool for differentiating meningiomas with atypical imaging characteristics from other tumors.

Radiation exposure is a potential concern with 320-row CT. However, a low-dose radiation protocol (adaptive iterative dose reduction) has recently become available. The effective radiation dose for this $4 \mathrm{D}$-CTA-CTP protocol amounted to $5.08-5.87 \mathrm{mSv}$, which is in line with the exposure of conventional CTP published in the literature [8]. Full coverage of the whole brain by 320-row CT is likely to deliver additional information that may justify increased radiation exposure. Finally, these new techniques based on 320-row CT can potentially be used to improve the differential diagnosis and preoperative assessment of cystic tumors.

\section{References}

1 Forutuna A, Ferrante L, Acqui M, Guglielmi G, Mastronardi L: Cystic meningiomas. Acta Neurochir (Wien) 1998;90:23-30.

2 Jain R: Perfusion CT imaging of brain tumors: an overview. AJNR Am J Neuroradiol 2011;32:1570-1577.

-3 Kremer S, Grand S, Remy C, Pasquir B, Benabid AL, Bracard S, Le Bas JF: Contribution of dynamic contrast MR imaging to the differentiation between dural metastasis and meningioma. Neuroradiol 2004;46:642648.

4 Liu M, Liu Y, Li X, Zhu S, Wu C: Cystic meningioma. J Clin Neurosci 2007;14:856-859.

5 Nauta HJ, Tucker WS, Horsey WJ, Bilvao JM, Gonsalves C: Xanthochromic cysts associated with meningioma. J Neurol Neurosurg Psychiatry 1979;42:529-535. 
Tabuchi et al.: Usefulness of 320-Row Area Detector Computed Tomography for the Diagnosis of Cystic Falx Meningioma

6 Siebert E, Bohner G, Masuhr F, Deuschle K, Diekmann S, Wiener E, Bauknecht HC, Klingebiel R: Neuroimaging by 320-row CT: is there a diagnostic benefit or is it just another scanner? A retrospective evaluation of 60 consecutive acute neurological patients. Neurol Sci 2010;31:585-593.

-7 Umansky F, Pappo I, Pizov G, Shalit M: Cystic changes in intracranial meningiomas. A review. Acta Neurochir (Wien) 1988;95:13-18.

8 Willems PW, Taeshineetanakul P, Schenk B, Brouwer PA, Terbrugge KG, Krings T: The use of 4D-CTA in the diagnostic work-up of brain arteriovenous malformations. Neuroradiol 2012;54:123-131.
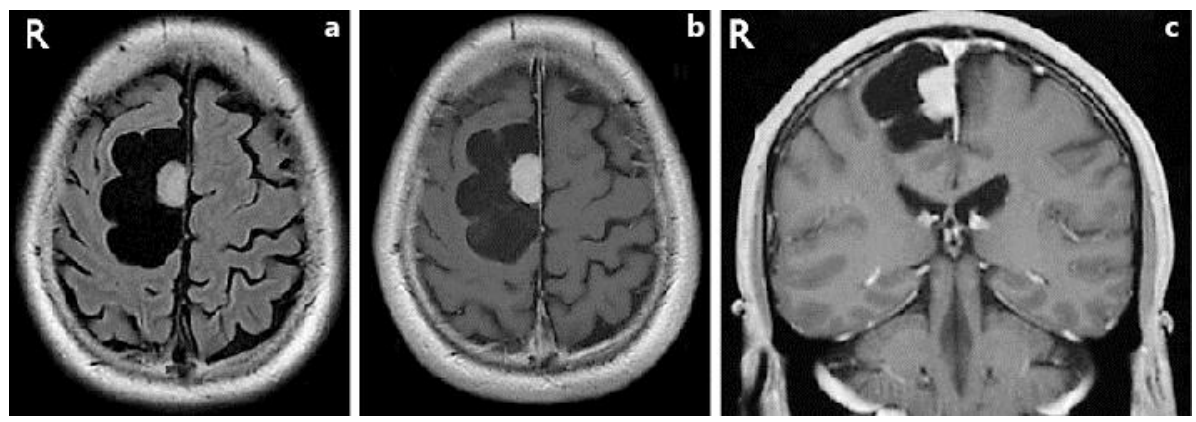

Fig. 1. a Fluid-attenuated inversion recovery (FLAIR) image shows a cyst within the right frontal lobe. The cystic component shows an intensity that is similar to cerebral spinal fluid. A small round mass can be seen, which appeared to be a mural nodule located at the cerebral falx. Perifocal edema was not evident. b Axial T1-weighted gadolinium-enhanced image shows that the tumor is enhanced homogeneously together with its attached dura mater, but the cyst wall is not enhanced. c Coronal T1-weighted gadolinium-enhanced image.
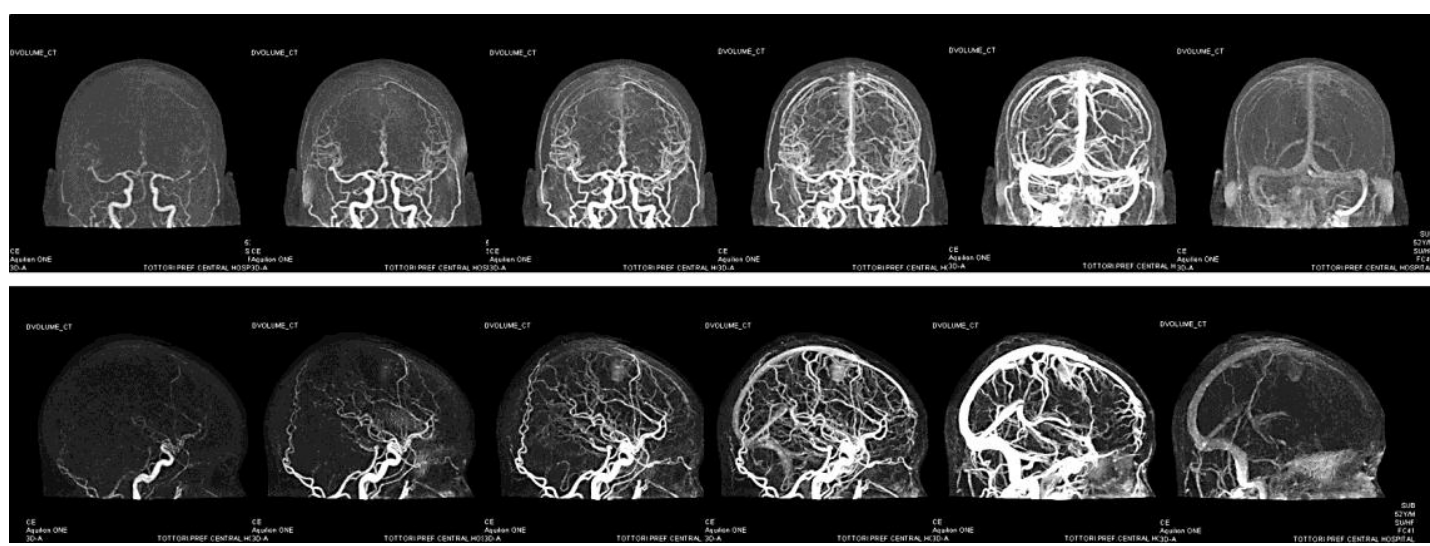

Fig. 2. Coronal serial images based on 4D-CTA (arterial - capillary - venous phase) show that middle meningeal artery feeding of the tumor nodule as well as contrast in the tumor becomes more pronounced at later time points (upper row). Sagittal serial images based on 4D-CTA (lower row). 


\section{Case Reports in Oncology}

\begin{tabular}{l|l}
\hline Case Rep Oncol 2013;6:362-366 \\
\hline DOI: $10.1159 / 000353929$ & $\begin{array}{l}\text { C 2013 S. Karger AG, Basel } \\
\text { www.karger.com/cro }\end{array}$ \\
\hline
\end{tabular}

Tabuchi et al.: Usefulness of 320-Row Area Detector Computed Tomography for the Diagnosis of Cystic Falx Meningioma
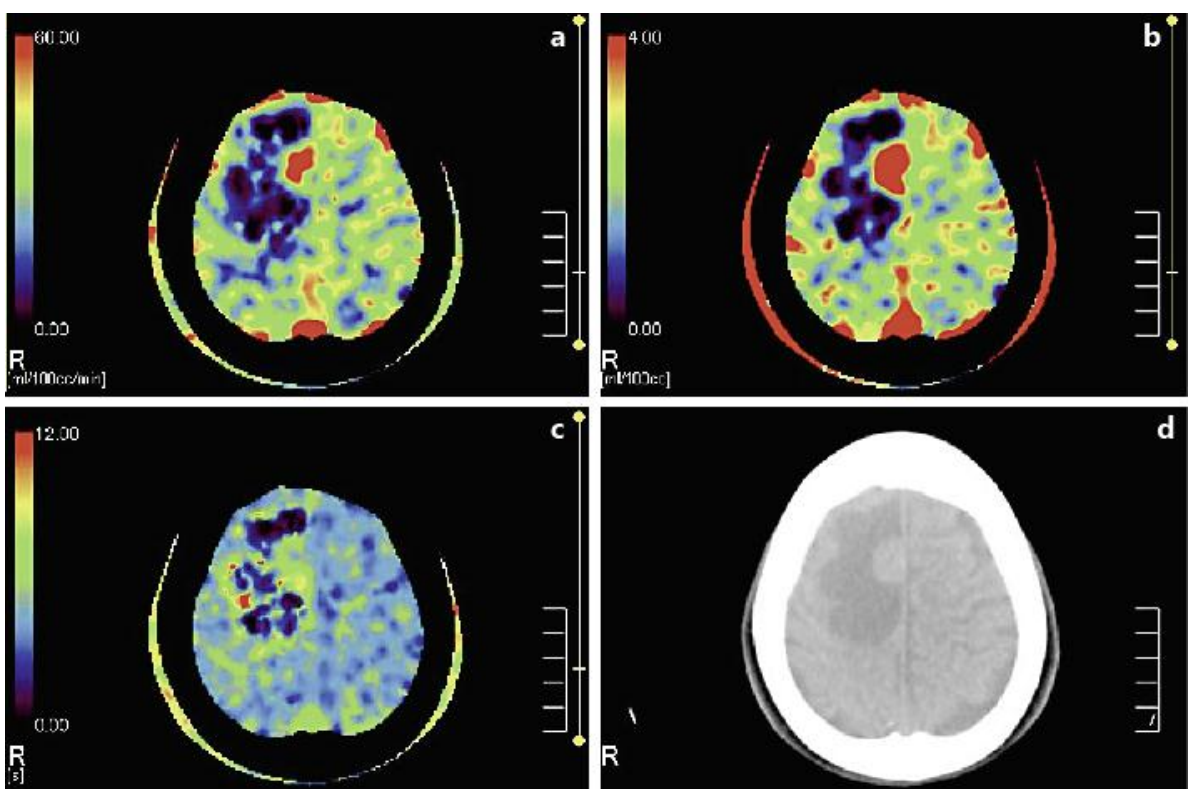

Fig. 3. a CBF map shows hyperperfusion of the tumor compared with the surrounding brain tissue. $\mathbf{b}$ CBV map shows increased blood volume in the tumor, suggesting marked hypervascularity. c MTT of the tumor was longer than that of the surrounding brain tissue. $\mathbf{d}$ Corresponding level of plain CT image. 